\title{
Comparison of different prognostic scores for risk stratification in septic patients arriving to the Emergency Department
}

\author{
Valeria Caramello, ${ }^{1}$ Valentina Beux, ${ }^{2}$ Alessandro Vincenzo De Salve, ${ }^{1}$ Alessandra Macciotta, ${ }^{3}$ Fulvio Ricceri, ${ }^{3}$ \\ Adriana Boccuzzi ${ }^{1}$ \\ ${ }^{1}$ Emergency Department and High Dependency Unit, AOU San Luigi Gonzaga, Orbassano (TO); ${ }^{2}$ University of Turin, Turin; \\ ${ }^{3}$ Department of Clinical and Biological Science, University of Turin, Turin, Italy
}

\begin{abstract}
We evaluated the prognostic performance of systemic inflammatory response syndrome (SIRS), sequential organ failure assessment (SOFA), quick-SOFA (qSOFA), modified early warning score (MEWS), lactates and procalcitonin in septic patients. Prospective study on adults with sepsis in the Emergency Department (ED). Area under the Receiver operator characteristic curve (AUC) was calculated to assess how scores predict mortality at 30 and 60 days (d) and upon admission to Intensive care unit (ICU). Among 469 patients, mortality was associated with higher SOFA, qSOFA, MEWS and lactates level. ICU admission was associated with higher SOFA, procalcitonin and MEWS. Prognostic performance for mortality were: SOFA AUC $30 \mathrm{~d} 0.76$ (0.69-0.81); $60 \mathrm{~d}$ 0.74 (0.68-0.79); qSOFAAUC $30 \mathrm{~d} 0.72$ (0.66-0.79); $60 \mathrm{~d} 0.73$ (0.67-0.78) and lactates AUC $30 \mathrm{~d} 0.71(0.60-0.82)$; 60d 0.65 (0.540.73). For the outcome ICU admission, procalcitonin had the highest AUC [0.66 (0.56-0.64], followed by SOFA [0.61 (0.54-0.69)] and MEWS [0.60 (0.53-0.67)]. SOFA, qSOFA and lactates assessment after arrival in the ED have a good performance in detecting patients at risk of mortality for sepsis. Procalcitonin is useful to select patients that will need ICU admission.
\end{abstract}

\section{Introduction}

Sepsis is defined as a life-threatening organ dysfunction that is caused by a dysregulated host response

Correspondence: Valeria Caramello, Emergency Department and High Dependency Unit MECAU, AOU San Luigi Gonzaga, regione Gonzole 10, 10043 Orbassano (TO), Italy. Tel.: +39.011.9026603.

E-mail: v.caramello@sanluigi.piemonte.it

Key words: Sepsis; sequential organ failure assessment (SOFA); quick SOFA; lactates; procalcitonin; mortality.

Contributions: VB, VC, ADS, and $\mathrm{AB}$ conceived the study, designed the protocol and obtained institutional review board approval. VB and VC undertook recruitment of patients, chart review, data collection and managed the data, including quality control. VC and $\mathrm{AB}$ supervised chart review and data collection. AM and FR provided statistical advice on study design and analyzed the data. VB and VC drafted the manuscript and all authors contributed substantially to its revision. VC takes responsibility for the paper as a whole.

Conflict of interests: the authors declare no conflict of interests.

Received for publication: 11 November 2019

Revision received: 11 March 2020.

Accepted for publication: 25 March 2020.

This work is licensed under a Creative Commons Attribution NonCommercial 4.0 License (CC BY-NC 4.0).

${ }^{\circ}$ Copyright: the Author(s), 2020

Licensee PAGEPress, Italy

Italian Journal of Medicine 2020; 14:79-87

doi:10.4081/itjm.2020.1232 to infection. ${ }^{1}$ It is a common cause of admission to the Emergency Department (ED), and it is essential to differentiate sepsis from an uncomplicated infection because sepsis can lead to multiple organ dysfunction syndrome and death. Early recognition of sepsis, ideally in the pre-hospital phase or during triage, can improve outcomes of these patients through corresponding interventions, which include timely fluids administration and appropriate antibiotics. However, because sepsis is a complex, heterogeneous disease, it is often difficult for clinicians to promptly identify patients with sepsis. ${ }^{2}$

There are no gold standard tests or diagnostic criteria to detect patients with sepsis. For more than two decades, the systemic inflammatory response syndrome (SIRS) criteria have been used in the diagnosis of sepsis. ${ }^{3}$ Researchers in several studies have reported controversies regarding the applicability of SIRS, and the SIRS criteria have also been criticized as a sepsis screening tool because of inadequate specificity and sensitivity. ${ }^{4}$ In 2016, the Society of Critical Care Medicine/European Society of Intensive Care Medicine task force released the Third International Consensus Definitions for Sepsis and Septic Shock (Sepsis-3) as a new definition for sepsis. ${ }^{5}$

Following this consensus statement, several studies tried to evaluate the prognostic value of SOFA and of the simplified version qSOFA in comparison with positive SIRS criteria for early identification of in-hospital mortality in patients with suspected infection. ${ }^{6-9}$ Other authors suggest the use of modified early warning score (MEWS), although not sepsis-specific, for 
the early recognition of patient at risk of clinical deterioration. ${ }^{10}$

Lactates and procalcitonin are the most validated and the most commonly used biomarkers for diagnosis and prognostic stratification of sepsis. ${ }^{11}$ The use of qSOFA in association with the assessment of venous lactates is suggested to increase qSOFA prognostic performance and it is shown that a lactate level above $2 \mathrm{mmol} / \mathrm{L}$ is associated with a worse outcome in terms of in-hospital mortality, admission to ICU and use of vasopressors. ${ }^{11}$ Similarly, patients with severe sepsis, septic shock and patients with sepsis that progresses towards a poor outcome had higher values of PCT compared to patients with a better prognosis. ${ }^{12}$

Aim of this study is to compare different tools (qSOFA, SIRS, SOFA, and MEWS) for the early diagnosis and prognostic stratification of septic patients arriving at the ED of San Luigi Gonzaga for sepsis.

\section{Materials and Methods}

A single-center study was performed: 510 patients ( $1.1 \%$ of the annual census) who subsequently visited the ED of San Luigi Gonzaga Orbassano (TO) from May 2018 to March 2019 for infections were evaluated. The study was approved by the Institutional Review Board.

We included patients who were 18 years or older and had a diffused infection of any severity, defined both by sepsis definition scores and by a clinical criteria, similarly to other studies. ${ }^{13,14}$ The inclusion criteria were: i) patients that visited the ED with a suspect of infection on a clinical or instrumental basis, associated with signs of SIRS; ii) patients that were identified as septic according to clinical judgment.

Patients were included only once regardless of the number of consultations at the ED. If patients consulted the ED more than once with a probable infection, the first consultation was selected. We excluded patients: i) who refused to participate; ii) with a lowacuity infection, defined by a localized infection without general symptoms and normal vital parameters; iii) in whom the positivity of the SIRS or qSOFA criteria is attributable in the first hypothesis to a non-infectious event (i.e. trauma, CAD, Stroke, acute pancreatitis). Patients who later needed surgical interventions, as part of their source control were included.

All data necessary for the SIRS (temperature, heart rate, blood pressure, respiratory rate, $\mathrm{PaCO}_{2}$, leukocytes), qSOFA (respiratory rate, systolic blood pressure, GCS), SOFA (Glasgow coma scale, altered mentation, blood pressure, respiratory rate, $\mathrm{PaO}_{2} / \mathrm{FIO}_{2}$, serum thrombocytes, bilirubin, lactate and creatinine and the use of vasopressor agents) and MEWS (respiratory rate, heart rate, systolic blood pressure, temperature, consciousness [Alert, Voice, Pain, Unconscious scale
(AVPU)], as well as general demographics such as age and gender were collected. Moreover, data about laboratory tests (lactates and procalcitonin assays) were collected when present. All data were collected in the first $12 \mathrm{~h}$ from arrival. MEWS was collected upon arrival from triage vitals; qSOFA was collected on arrival $\left(\mathrm{t}_{0}\right)$, $6 \mathrm{~h}$ from arrival $\left(\mathrm{t}_{6}\right)$ and $12 \mathrm{~h}\left(\mathrm{t}_{12}\right)$ from arrival. This timing was chosen because the right time of qSOFA assessment is still controversial. SOFA was calculated at $12 \mathrm{~h}$ when laboratory tests were available.

Sepsis based on the SIRS criteria (Sepsis-1 definition) was defined as a probable infection combined with a SIRS score of $\geq 2$ points. ${ }^{9}$ Sepsis based on the Sepsis-3 criteria was defined as infection with a SOFA score $\geq 2$ points from the baseline. ${ }^{1}$ Furthermore, we assessed the use of qSOFA instead of SOFA in this same definition, with the standard cut-off of qSOFA $\geq 2$ points. ${ }^{1}$ MEWS $\geq 5$ points was previously found to be predictive of mortality in septic patients, thus we applied the same cut-off. ${ }^{10}$ Lactate levels over 2 and procalcitonin levels over 2 are considered biomarkers of severe sepsis. ${ }^{11,12}$

The evaluation of the variables and the scores did not interfere with the current clinical practice; the attending physician was unaware of the results of the scores calculated. ${ }^{1,9}$

We prospectively evaluated the following outcomes: 30-day mortality, 60-day mortality and admission to a higher level of care wards [Intensive Care Unit (ICU) and High Dependency Unit (HDU)]. Furthermore, we evaluated secondary ICU/HDU admission (for deterioration of the clinical condition after admission to the general ward). When a patient was admitted both to ICU or to HDU we considered admission (primary or secondary) in high-level-of care ward (ICU-adm). The decision for ICU/HDU admission followed the usual clinical practice, institutional guidelines and local policies.

Outcomes were derived by reviewing the hospital digital records and by a phone call to collect information after discharge.

Data were described using means and standard deviations (SD) for continuous variables, medians and interquartile ranges (IQR) for discrete variables, absolute frequencies and percentages for categorical and qualitative variables. The Shapiro-Wilk test ${ }^{15}$ was used to test the normality of the distribution of quantitative variables, and since most of the distributions violated the normality assumption, non-parametric tests were conducted. We evaluated the number of cases defined as septic according to the different sepsis definitions. We compared the results of the scores between subgroups of different outcomes (survivors $v s$ non-survivors; admitted in ICU or HDU $v s$ admitted in a regular ward) using Wilcoxon rank sum test ${ }^{16}$ or Chi square test, ${ }^{17}$ as appropriate. 
Receiver operator characteristic (ROC) curves and the area under the ROC curve (area under the curve AUC) were calculated to determine how clinical scores and biomarkers (qSOFA, SOFA, SIRS, lactates, and procalcitonin) predict the primary endpoints. We calculated confidence interval at 95\% (CI 95\%) for all AUCs to assess if the null hypothesis $(\mathrm{AUC}=0.50)$ was included in the interval. De Long test was performed to compare correlated ROC curves. For each combination of clinical score and outcome parameter, sensitivity (Se), specificity (Sp), positive predictive value (PPV), negative predictive value (NPV), positive likelihood ratio $(\mathrm{LH}+)$ and negative likelihood ratio $(\mathrm{LH}-)$ were computed according to the standard cut-off.

All tests were two-tailed and a P-value of 0.05 or less was considered statistically significant.

All statistical analyses were carried out using SAS Software Version 9.2 ${ }^{18}$ and R Version 3.5.2 (Eggshell Igloo, 2018-12-20). ${ }^{19}$

\section{Results}

A total of 510 patients were enrolled during the recruitment period. 41 patients were lost to follow up, leaving 469 patients included for the final analysis. The median age was 73 years (range: 61-81), 325 (69\%) pa- tients were older than 65 years. There were 267 (57\%) male patients and 202 (43\%) female patients.

$17 \%$ of patients were admitted to hospital for infection in the month before the recruitment, $8 \%$ of patients were admitted for other reasons in the month before the recruitment.

$28 \%$ of our cohort was concurrently suffering from neoplasm, $23 \%$ presented diabetes, $24 \%$ had heart failure or coronary artery disease (CAD) or stroke events in the past and $13 \%$ of patients were immunosuppressed.

Baseline characteristics are summarized in Table 1.

Among the data we collected, Respiratory Rate was missing in nearly $10 \%$ of cases, thus we assume that it was in the normal range for computing qSOFA and MEWS at $\mathrm{t}_{0}$. The other variables in the study were not affected by missing values except for lactates and procalcitonin.

At follow-up, all patients received antibiotic therapy, $347(74 \%)$ patients received fluid therapy, 12 $(3 \%)$ patients in our cohort needed vasopressors administration.

The overall admittance rate was $79 \%$ (371); in this group, 68 patients were admitted to the ICU (4) or HDU (64), respectively. 15 more patients were subsequently transferred to ICU for clinical deterioration (Secondary ICU).

Table 1. The results of the scores in the overall population and in the group of patients according to their outcome. Values are expressed as median [interquartile range].

\begin{tabular}{|c|c|c|c|c|}
\hline & Overall & Alive at 60 days & Dead at 60 days & $\mathbf{P}$ \\
\hline Age years & $\begin{array}{c}69.69 \pm 16.4 \\
73[61-81]\end{array}$ & $\begin{array}{l}67.4 \pm 16.7 \\
71[57-80]\end{array}$ & $\begin{array}{c}79.11 \pm 11.01 \\
80[75-87]\end{array}$ & $<0.001^{*}$ \\
\hline Gender $\mathrm{M} / \mathrm{F}$ & $267 / 202$ & $206 / 171$ & $61 / 31$ & $0.048^{\S}$ \\
\hline SIRS & $2[1-3]$ & $2[1-3]$ & $2[1-3]$ & $0.46^{*}$ \\
\hline SOFA & $2[1-4]$ & $2[1-3]$ & $4[2-7]$ & $<0.001^{*}$ \\
\hline qSOFA $t_{0}$ & $1[0-1]$ & $0[0-1]$ & $1[1-2]$ & $<0.001^{*}$ \\
\hline $\mathrm{qSOFA} \mathrm{t}_{6}$ & $0[0-1]$ & $0[0-1]$ & $1[0-2]$ & $<0.001^{*}$ \\
\hline qSOFA $_{12}$ & $0[0-1]$ & $0[0-1]$ & $1[0-2]$ & $<0.001^{*}$ \\
\hline MEWS & $3[1-4]$ & $2[1-4]$ & $4[1.5-5]$ & $0.0034^{*}$ \\
\hline Lactates mmol/L & $\begin{array}{c}2.26 \pm 2.33 \\
1.5[1-2.5] \\
(\mathrm{n}=141)\end{array}$ & $\begin{array}{c}1.8 \pm 2 \\
1.4[0.9-2.1] \\
(\mathrm{n}=101)\end{array}$ & $\begin{array}{l}3.27 \pm 2.8 \\
2.25[1-5] \\
(n=40)\end{array}$ & $0.005 *$ \\
\hline $\mathrm{PCT} n \mathrm{~g} / \mathrm{mL}$ & $\begin{array}{c}5.37 \pm 14 \\
0.6[0.18-3.29] \\
(n=302)\end{array}$ & $\begin{array}{c}4.26 \pm 11 \\
0.49[0.17-2.57] \\
(\mathrm{n}=247)\end{array}$ & $\begin{array}{c}10.3 \pm 23 \\
1.06[0.23-7.03] \\
(\mathrm{n}=55)\end{array}$ & $0.038^{*}$ \\
\hline $\begin{array}{l}\text { Recent }(<1 \text { month }) \\
\text { hospitalization } \\
\text { for infectionfor other causes }\end{array}$ & $\begin{array}{c}114(24 \%) \\
78 \\
36\end{array}$ & $\begin{array}{l}93(24 \%) \\
63 \\
30\end{array}$ & $\begin{array}{c}21(22 \%) \\
15 \\
6\end{array}$ & $0.7^{\S}$ \\
\hline $\mathrm{n}$ & 469 & 377 & 92 & \\
\hline
\end{tabular}

Comparisons were made according to Wilcoxon rank sum test* and Chi square test $\$$. $\mathrm{P}<0.05$ are in italic. The number of cases (n) for lactates and procalcitonin (PCT) are reported in brackets. SIRS, systemic inflammatory response syndrome; SOFA, sequential organ failure assessment; qSOFA, quick SOFA; MEWS, modified early warning score; PCT, procalcitonin. 
$98(21 \%)$ were discharged home or to outpatient clinics from the ED.

In our sample, 92 patients (19.6\%) died: $90 \%$ of them (83) died during their stay in the hospital, 14 $(15 \%)$ died in the Emergency Department and $9(10 \%)$ patients died after being discharged from our hospital.

Examining the final ED diagnosis 451 patients were defined by the ED physician as having sepsis, 18 patients as having septic shock.

We identified 325 patients $(69 \%)$ as having sepsis using the Sepsis-1 definition, and 292 patients (62\%) using the Sepsis-3 definition with $S O F A \geq 2$. Using the qSOFA, 89 (19\%) fulfilled the definition criteria at the time of arrival $\left(\mathrm{t}_{0}\right), 69(15 \%)$ after 6 hours $\left(\mathrm{t}_{6}\right)$ and 62 $(13 \%)$ after 12 hours $\left(t_{12}\right)$. Considering the highest one out of the three qSOFA (qSOFA Overall), 123 patients (26\%) fulfilled the definition criteria. A total of $72 \mathrm{pa}-$ tients $(16 \%)$ were not picked up by any criteria.

Sepsis-related organ dysfunction according to the Sepsis-1 criteria was present in 127 patients out of 325 patients (39\%).

Only 21 patients (4\%) presented with septic shock according to the Sepsis-1 definition, and 13 patients (3\%) presented with septic shock according to the Sepsis-3 definitions.

The relationship between study groups according to Sepsis-1 and Sepsis-3 definitions are illustrated graphically in Figure 1.

Median MEWS was 3 [IQR 1-4], with 87 (18\%)

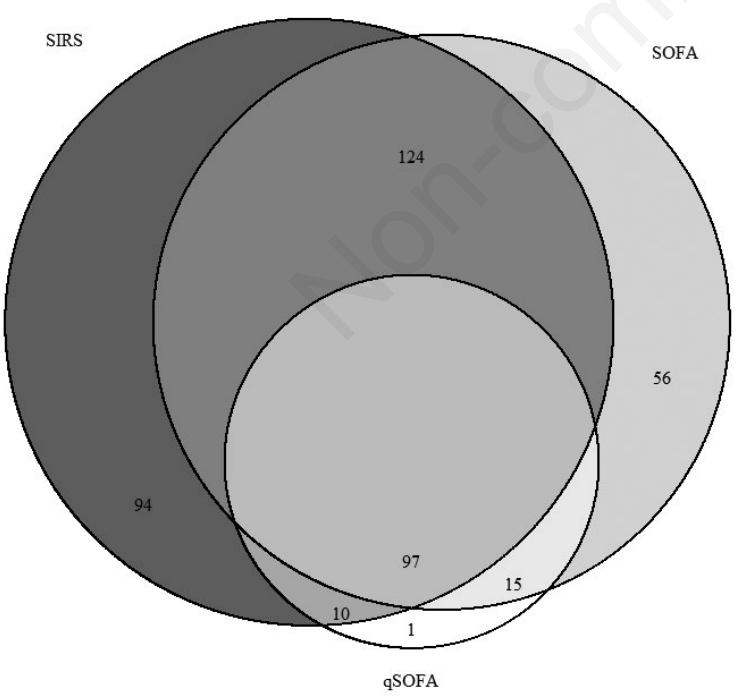

Figure 1. It describes graphically the proportion of patients included in each diagnostic definition of sepsis according to the different scores. Sepsis based on the SIRS criteria (Sepsis-1) ${ }^{3}$ was defined as a probable infection combined with a SIRS score of $\geq 2$ points (in black). Sepsis based on the Sepsis-3 criteria1 was defined as infection with a SOFA score $\geq 2$ points (in grey) and a qSOFA $\geq 2$ points (in white). patients of our sample over the standard cut-off (MEWS $\geq 5$ ). ${ }^{10}$

Serum lactates were assessed in $141(30 \%)$ patients, out of them only $52(37 \%)$ patients had a lactate measurement $\geq 2 \mathrm{mmol} / \mathrm{L} .^{11}$

Procalcitonin was assessed in 302 (64\%) patients, out of them only $98(32 \%)$ had a procalcitonin measurement $\geq 2 \mathrm{ng} / \mathrm{mL}$. $^{12}$

Afterwards we evaluated the prognostic value of the scores with regards to the outcomes.

\section{Mortality}

Thirty- and 60-day mortalities were $15.8 \%$ (74) and $19.6 \%$ (92), respectively. Patients who died were significantly older and got statistically significant higher qSOFA, SOFA and MEWS scores compared to patients who survived. Conversely, SIRS score did not show a statistically significant difference between non-survivors and survivors' groups, as shown in Table 1. Lactates levels were significantly higher in patients who died in comparison with the ones that survived; a similar trend was shown for procalcitonin although it did not reach the statistical significance.

An AUC curve for the prediction of death/ mortality was constructed with new and former definitions of sepsis, namely SOFA, qSOFA, SIRS, MEWS, lactates and procalcitonin measurements. The highest AUCs were for the SOFA score [30 days 0.76 (0.69$0.81) ; 60$ days $0.74(0.68-0.79)]$ and qSOFA score [ 30 days $0.72(0.65-0.79)$; 60 days $0.73(0.66-0.78)]$ followed by lactates [30 days 0.71 (0.60-0.82); 60 days $0.65(0.54-0.76)]$ as shown in Figure 2.

Prognostic performances of SOFA, qSOFA, SIRS, MEWS, lactates and procalcitonin according to the standard cut-offs to predict 30- and 60-day mortality are reported in Table 2.

Comparison of ROC curves showed that the performance of qSOFA and SOFA was not significantly different for the prediction of mortality at 30 and 60 days $(\mathrm{P}=0.34$ and $\mathrm{P}=0.76$ respectively); both performed significantly better than SIRS (qSOFA at $30 \mathrm{~d}$ and $60 \mathrm{~d}$ $\mathrm{P}<0.001$, SOFA at $30 \mathrm{~d}$ and $60 \mathrm{~d} \mathrm{P}<0.001)$. MEWS was significantly superior to $\operatorname{SIRS}(\mathrm{P}=0.01$ at $30 \mathrm{~d}$ and $\mathrm{P}<0.01$ at $60 \mathrm{~d})$ but inferior to SOFA $(\mathrm{P}<0.001$ at $30 \mathrm{~d}$ and $60 \mathrm{~d})$ and qSOFA $(\mathrm{P}<0.001$ at $30 \mathrm{~d}$ and $60 \mathrm{~d})$.

Table 2 shows the characteristics of the scores for the prediction of 30-day and 60-day mortality. Se, Sp, PPV, NPV, LH+, and LH- were calculated for the existing cut-offs.

\section{Intensive Care Unit/High Dependence Unit admission}

A total of 68 patients (14.5\%) of our cohort were admitted to the Intensive Care Unit or High Dependency Unit, while 15 (3\%) were admitted to the Inten- 
sive Care Unit or High Dependency Unit after a median of 9 days in a general ward (secondary ICU admission).

SOFA, qSOFA score at $t_{12}$, procalcitonin and MEWS score were statistically significant predictors of ICU/HDU admission, showing higher values in ICU patients. Details are described in Table 3.

Procalcitonin, SOFA and MEWS were the only effective predictors of ICU admission with the following AUC: PCT 0.66 (0.56-0.64), MEWS 0.60 (0.53-0.67) and SOFA $0.61(0.54-0.69)$, respectively (details in Table 2).

In the supplementary material, the ROC curves for each parameter included in the study are represented and described individually for each outcome.

Table 2 shows the characteristics of the scores for the prediction of ICU admission. Se, Sp, PPV, NPV, $\mathrm{LH}+$, and $\mathrm{LH}-$ were calculated for the existing cut-offs.

\section{Discussion}

Sepsis is a systemic disease with variable clinical presentations but without a gold standard for a definitive diagnosis. This prospective study included patients with suspected infection of all severities and considered outcomes in the short and medium term, aiming to find the best tool for the early diagnosis and prognostic stratification of septic patients in the ED.

Sepsis-1 criteria, ${ }^{9}$ namely the presence of 2 or more SIRS criteria associated with infection, identified most patients in the at-risk population $(69 \%)$, followed by Sepsis- $3^{1}$ (namely SOFA of 2 or more) $(62 \%)$. The use of qSOFA dramatically reduced the number of patients classified as septic (26\%). There was a partial overlap between the diagnostic criteria, with half of the septic patients identified by both SIRS and SOFA and half of the patients that are classified in the same category by SOFA and qSOFA. Many cases showed discordant results at the three scoring systems, similarly to previous studies. ${ }^{20}$

These results confirmed that scores have limitations in diagnosing this complex heterogeneous situation as sepsis is: SIRS criteria are more sensitive, SOFA is a complete and specific tool and qSOFA is easy to perform but is not completely concordant with SOFA. Moreover, we lack a gold standard to compare the scores with: we used clinical diagnosis at the end of ED stay and antibiotic administration to confirm that our population was defined as septic.

On the other hand, the most recent sepsis definition and guidelines (Sepsis-3) ${ }^{1}$ strongly focus on identifying patients with a poor prognosis and suggest the use of scores for risk stratification in order to select candidates for early intensive treatment. SOFA, qSOFA and lactates levels confirmed to be accurate in predicting mortality. Our population showed a mortality rate of nearly $19 \%$, in line with other studies in different countries. ${ }^{21,22}$ Having an elevated SOFA, qSOFA, MEWS score, elevated lactates, and procalcitonin levels it was related to a worse prognosis. SOFA, qSOFA and MEWS were found to be accurate, with qSOFA being the most specific and SOFA the most sensitive tools. Small differences in sensitivity and specificity were observed when comparing the characteristics of the tools to evaluate short-term and medium-term mortality. While SOFA was confirmed to be like SIRS in patient identification and superior to SIRS ${ }^{5,22-24}$ in prediction of mortality outcome, it requires complete blood test analysis. SOFA's variables could be unavailable in specific settings and this could create delays in patient treatment. The prospective nature of our study, in this respect, was useful to avoid missing data.

qSOFA is simplified in comparison with SOFA, but in 9 to $51 \%$ of cases $^{23}$ is described to suffer from
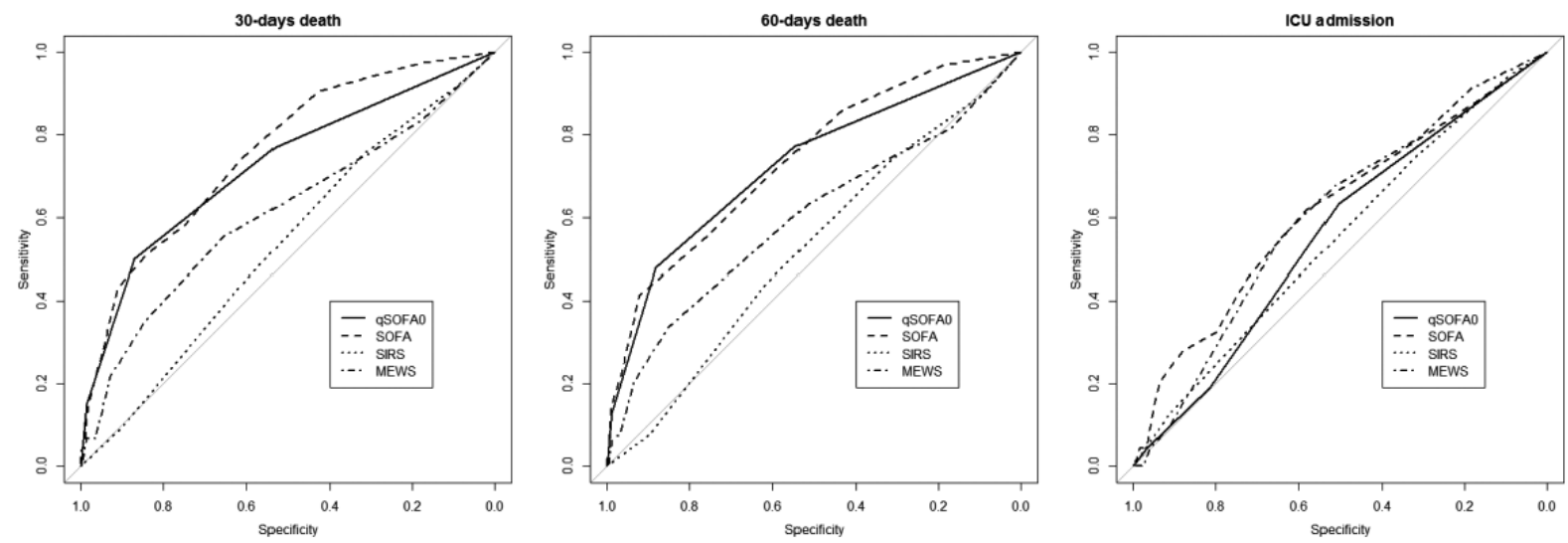

Figure 2. It compares, for each of the outcomes in the study, the ROC curves for the parameters included in study. qSOFA is in full-line, SOFA in dashed line, SIRS in dotted line, MEWS in mixed line. 
Table 2. The characteristics of the scores for the outcome mortality at $\mathbf{3 0}$ and $\mathbf{6 0}$ days and for Intensive Care Unit admission.

\begin{tabular}{|c|c|c|c|}
\hline & Outcome death 30 days & Outcome death 60 days & ICU admission \\
\hline SIRS & $\begin{array}{l}\text { Se } 0.76(0.64,0.85) \\
\text { Sp } 0.32(0.27,0.36) \\
\text { PPV } 0.17(0.13,0.22) \\
\text { NPV } 0.87(0.81,0.92) \\
\text { LH+ } 1.11(0.96,1.28) \\
\text { LH- } 0.77(0.50,1.18) \\
\text { AUC } 0.535(0.467,0.603) \\
\text { Cut-off } 2\end{array}$ & $\begin{array}{l}\text { Se } 0.74(0.64,0.83) \\
\text { Sp } 0.32(0.27,0.37) \\
\text { PPV } 0.21(0.17,0.26) \\
\text { NPV } 0.83(0.76,0.89) \\
\text { LH+ } 1.08(0.94,1.24) \\
\text { LH- } 0.83(0.57,1.20) \\
\text { AUC } 0.524(0.461,0.587) \\
\text { Cut-off } 2\end{array}$ & $\begin{array}{l}\text { Se } 0.75(0.63,0.85) \\
\text { Sp } 0.32(0.27,0.36) \\
\text { PPV } 0.16(0.12,0.20) \\
\text { NPV } 0.88(0.82,0.93) \\
\text { LH+ } 1.09(0.94,1.28) \\
\text { LH- } 0.79(0.51,1.23) \\
\text { AUC } 0.548(0.476,0.619) \\
\text { Cut-off } 2\end{array}$ \\
\hline SOFA & $\begin{array}{l}\text { Se } 0.91(0.81,0.96) \\
\text { Sp } 0.43(0.38,0.48) \\
\text { PPV } 0.23(0.18,0.28) \\
\text { NPV } 0.96(0.92,0.98) \\
\text { LH+ } 1.59(1.42,1.77) \\
\text { LH- } 0.22(0.11,0.45) \\
\text { AUC } 0.758(0.699,0.816) \\
\text { Cut-off } 2\end{array}$ & $\begin{array}{l}\text { Se } 0.86(0.77,0.92) \\
\text { Sp } 0.43(0.38,0.49) \\
\text { PPV } 0.27(0.22,0.33) \\
\text { NPV } 0.93(0.88,0.96) \\
\text { LH+ } 1.52(1.34,1.71) \\
\text { LH- } 0.33(0.19,0.55) \\
\text { AUC } 0.738(0.681,0.795) \\
\text { Cut-off } 2\end{array}$ & $\begin{array}{l}\text { Se } 0.74(0.61,0.83) \\
\text { Sp } 0.40(0.35,0.44) \\
\text { PPV } 0.17(0.13,0.22) \\
\text { NPV } 0.90(0.84,0.94) \\
\text { LH+ } 1.22(1.03,1.43) \\
\text { LH- } 0.67(0.44,1.01) \\
\text { AUC } 0.617(0.541,0.692) \\
\text { Cut-off } 2\end{array}$ \\
\hline qSOFA $t_{0}$ & $\begin{array}{l}\text { Se } 0.50(0.38,0.62) \\
\text { Sp } 0.87(0.83,0.90) \\
\text { PPV } 0.42(0.32,0.53) \\
\text { NPV } 0.90(0.87,0.93) \\
\text { LH+ } 3.87(2.75,5.46) \\
\text { LH- } 0.57(0.46,0.72) \\
\text { AUC } 0.725(0.659,0.79) \\
\text { Cut-off } 2\end{array}$ & $\begin{array}{l}\text { Se } 0.48(0.37,0.58) \\
\text { Sp } 0.88(0.85,0.91) \\
\text { PPV } 0.50(0.39,0.61) \\
\text { NPV } 0.87(0.84,0.91) \\
\text { LH+ } 4.10(2.89,5.82) \\
\text { LH- } 0.59(0.48,0.72) \\
\text { AUC } 0.728(0.669,0.786) \\
\text { Cut-off } 2\end{array}$ & $\begin{array}{l}\text { Se } 0.19(0.11,0.30) \\
\text { Sp } 0.81(0.77,0.85) \\
\text { PPV } 0.15(0.08,0.24) \\
\text { NPV } 0.86(0.82,0.89) \\
\text { LH+ } 1.03(0.61,1.76) \\
\text { LH- } 0.99(0.88,1.12) \\
\text { AUC } 0.558(0.492,0.625) \\
\text { Cut-off } 2\end{array}$ \\
\hline $\mathrm{qSOFA} \mathrm{t}_{6}$ & $\begin{array}{l}\text { Se } 0.43(0.31,0.56) \\
\text { Sp } 0.89(0.85,0.92) \\
\text { PPV } 0.43(0.31,0.55) \\
\text { NPV } 0.89(0.86,0.92) \\
\text { LH+ } 3.95(2.64,5.92) \\
\text { LH- } 0.64(0.52,0.79) \\
\text { AUC } 0.712(0.642,0.782) \\
\text { Cut-off } 2\end{array}$ & $\begin{array}{l}\text { Se } 0.39(0.29,0.51) \\
\text { Sp } 0.90(0.86,0.93) \\
\text { PPV } 0.49(0.36,0.61) \\
\text { NePV } 0.86(0.82,0.89) \\
\text { LH+ } 3.81(2.52,5.74) \\
\text { LH- } 0.68(0.57,0.81) \\
\text { AUC } 0.714(0.652,0.775) \\
\text { Cut-off } 2\end{array}$ & $\begin{array}{l}\text { Se } 0.26(0.16,0.39) \\
\text { Sp } 0.86(0.82,0.89) \\
\text { PPV } 0.24(0.14,0.36) \\
\text { NPV } 0.87(0.83,0.91) \\
\text { LH+ } 1.86(1.14,3.04) \\
\text { LH- } 0.86(0.74,1.00) \\
\text { AUC } 0.576(0.501,0.651) \\
\text { Cut-off } 2\end{array}$ \\
\hline qSOFA $t_{12}$ & $\begin{array}{l}\text { Se } 0.46(0.33,0.59) \\
\text { Sp } 0.91(0.87,0.94) \\
\text { PPV } 0.48(0.35,0.62) \\
\text { NPV } 0.90(0.87,0.93) \\
\text { LH+ 5.11 }(3.30,7.91) \\
\text { LH- } 0.59(0.47,0.75) \\
\text { AUC } 0.698(0.621,0.775) \\
\text { Cut-off } 2\end{array}$ & $\begin{array}{l}\text { Se } 0.40(0.29,0.52) \\
\text { Sp } 0.92(0.88,0.94) \\
\text { PPV } 0.53(0.40,0.67) \\
\text { NPV } 0.86(0.82,0.90) \\
\text { LH+ } 4.74(3.02,7.45) \\
\text { LH- } 0.65(0.54,0.79) \\
\text { AUC } 0.693(0.625,0.761) \\
\text { Cut-off } 2\end{array}$ & $\begin{array}{l}\text { Se } 0.23(0.13,0.36) \\
\text { Sp } 0.87(0.83,0.90) \\
\text { PPV } 0.23(0.13,0.36) \\
\text { NPV } 0.87(0.83,0.91) \\
\text { LH+ } 1.78(1.03,3.09) \\
\text { LH- } 0.88(0.76,1.03) \\
\text { AUC } 0.592(0.516,0.669) \\
\text { Cut-off } 2\end{array}$ \\
\hline MEWS & $\begin{array}{l}\text { Se } 0.35(0.24,0.47) \\
\text { Sp 0.85 }(0.81,0.88) \\
\text { PPV } 0.30(0.21,0.41) \\
\text { NPV } 0.87(0.84,0.91) \\
\text { LH+ } 2.28(1.55,3.35) \\
\text { LH- } 0.77(0.65,0.91) \\
\text { AUC } 0.61(0.533,0.687) \\
\text { Cut-off } 5\end{array}$ & $\begin{array}{l}\text { Se } 0.34(0.24,0.44) \\
\text { Sp } 0.85(0.81,0.89) \\
\text { PPV } 0.36(0.26,0.47) \\
\text { NPV } 0.84(0.80,0.88) \\
\text { LH+ } 2.27(1.56,3.30) \\
\text { LH- } 0.78(0.67,0.91) \\
\text { AUC } 0.597(0.526,0.667) \\
\text { Cut-off } 5\end{array}$ & $\begin{array}{l}\text { Se } 0.25(0.15,0.37) \\
\text { Sp } 0.82(0.78,0.86) \\
\text { PPV } 0.20(0.12,0.29) \\
\text { NPV } 0.87(0.83,0.90) \\
\text { LH+ } 1.43(0.90,2.27) \\
\text { LH- } 0.91(0.79,1.05) \\
\text { AUC } 0.604(0.534,0.674) \\
\text { Cut-off } 5\end{array}$ \\
\hline Lactates $(\mathrm{n}=141)$ & $\begin{array}{l}\text { Se } 0.62(0.44,0.78) \\
\text { Sp } 0.71(0.61,0.79) \\
\text { PPV } 0.40(0.27,0.55) \\
\text { NPV } 0.85(0.76,0.92) \\
\text { LH+ } 2.13(1.43,3.17) \\
\text { LH- } 0.54(0.35,0.84) \\
\text { AUC } 0.717(0.607,0.826) \\
\text { Cut-off } 2\end{array}$ & $\begin{array}{l}\text { Se } 0.55(0.38,0.71) \\
\text { Sp } 0.70(0.60,0.79) \\
\text { PPV } 0.42(0.29,0.57) \\
\text { NPV } 0.80(0.70,0.88) \\
\text { LH+ } 1.85(1.23,2.79) \\
\text { LH- } 0.64(0.44,0.92) \\
\text { AUC } 0.652(0.54,0.763) \\
\text { Cut-off } 2\end{array}$ & $\begin{array}{l}\text { Se } 0.48(0.30,0.67) \\
\text { Sp } 0.66(0.57,0.75) \\
\text { PPV } 0.29(0.17,0.43) \\
\text { NPV } 0.82(0.72,0.89) \\
\text { LH+ } 1.44(0.92,2.25) \\
\text { LH- } 0.78(0.54,1.12) \\
\text { AUC } 0.577(0.464,0.69) \\
\text { Cut-off } 2\end{array}$ \\
\hline PCT $(n=302)$ & $\begin{array}{l}\text { Se } 0.40(0.25,0.56) \\
\text { Sp } 0.69(0.63,0.74) \\
\text { PPV } 0.17(0.10,0.26) \\
\text { NPV } 0.87(0.82,0.92) \\
\text { LH+ } 1.26(0.84,1.91) \\
\text { LH- } 0.88(0.68,1.14) \\
\text { AUC } 0.605(0.512,0.698) \\
\text { Cut-off } 2\end{array}$ & $\begin{array}{l}\text { Se } 0.38(0.25,0.52) \\
\text { Sp } 0.69(0.63,0.75) \\
\text { PPV } 0.21(0.14,0.31) \\
\text { NPV } 0.83(0.77,0.88) \\
\text { LH+ } 1.22(0.83,1.80) \\
\text { LH- } 0.90(0.72,1.12) \\
\text { AUC } 0.589(0.505,0.674) \\
\text { Cut-off } 2\end{array}$ & $\begin{array}{l}\text { Se } 0.60(0.45,0.74) \\
\text { Sp } 0.73(0.67,0.78) \\
\text { PPV } 0.30(0.21,0.40) \\
\text { NPV } 0.91(0.86,0.94) \\
\text { LH+ } 2.22(1.64,3.02) \\
\text { LH- } 0.54(0.38,0.78) \\
\text { AUC } 0.656(0.564,0.748) \\
\text { Cut-off } 2\end{array}$ \\
\hline
\end{tabular}

Sensitivity (Se), specificity (Sp), Positive and negative predictive value (PPV and NPV) and Positive and negative likelihood ratio (LH+ and LH-) are calculated for the existing cutoff. Area Under the Curve is displayed. Confidence interval at 95\% (CI95\%) is described in brackets. SIRS, systemic inflammatory response syndrome; SOFA, sequential organ failure assessment; qSOFA, quick SOFA; MEWS, modified early warning score; PCT, procalcitonin; ICU, Intensive Care Unit and High Dependency Unit. 
missing pieces of information about the neurological status and from the absence of the record for respiratory rate. When no report was found on the respiratory status (less than $10 \%$ of cases) we assigned the normal score to the item, similarly to previous studies. We found information about neurological status in all the patients. In our study qSOFA was more specific but less sensitive than SOFA for all the outcomes but performed equally well.

The simplified qSOFA which was proposed for bedside use in the ED is suggested to be repeated and considered by its variance in time. ${ }^{1}$ In our study qSOFA was calculated upon arrival and after 6 and 12 $\mathrm{h}$ : the accuracy of qSOFA in predicting mortality was similar upon arrival and in the following serial intervals; the accuracy in predicting mortality and ICU admission increased in serial measurements. In this setting, serial qSOFA could be used to evaluate the dynamic and evolving characteristics of sepsis, in accordance with other authors findings. ${ }^{23}$

These results are in line with other studies performed in ICU, general wards and ED settings in Europe, USA, Africa and Australia ${ }^{20,24-26}$ and with a recent meta-analysis. ${ }^{27}$

Most patients of our population were admitted, less than $20 \%$ of admissions were to ICU or HDU units, with an additional $3 \%$ of patients admitted to a regular ward and then transferred to intensive care unit for physiological deterioration. Globally the accuracy of the scores was lower in predicting the need for in- tensive care, but this result could be biased by the low number of cases. Moreover, ICU admittance is affected by age and co-morbid conditions (like DNR status) and it is not only determined by sepsis severity.

MEWS was not originally designed for recognition of sepsis patients and is meant to evaluate the evolving severity of any illness, not only sepsis. ${ }^{10,28,29}$ We do not support the use of MEWS alone for the diagnosis of sepsis, but our data support its use in selecting patients that will need ICU admission.

Procalcitonin, as a marker of the most serious infections, has a role in evaluating the burden of the disease and the ICU admission, and should be used in association with the above-described scores. Although a perfect diagnostic tool is a pure utopia and all the studies on septic patients are biased by the lack of a gold standard, the inclusion in any definition proved again to be reductive for a complex and pleomorphic syndrome where the clinical gestalt associated with some diagnostic tests is probably still the best decision-making pathway.

We chose to evaluate outcomes that are crucial for the decision process in the ED and that are under the direct responsibility of the Emergency Physician in the first $12 \mathrm{~h}$ from arrival: short term mortality, admission to a high level of care wards (both ICU and HDU). Medium-term mortality is partially related to the global burden of sepsis on functional disability in short-term survivors that can lead to further illnesses, further hospitalizations and long-term death. This can

Table 3. Median values of the scores in patients admitted to Intensive Care Unit and High Dependency Unit (ICU), admitted to the regular ward and admitted to ICU after the deterioration of clinical conditions during hospitalization (secondary ICU).

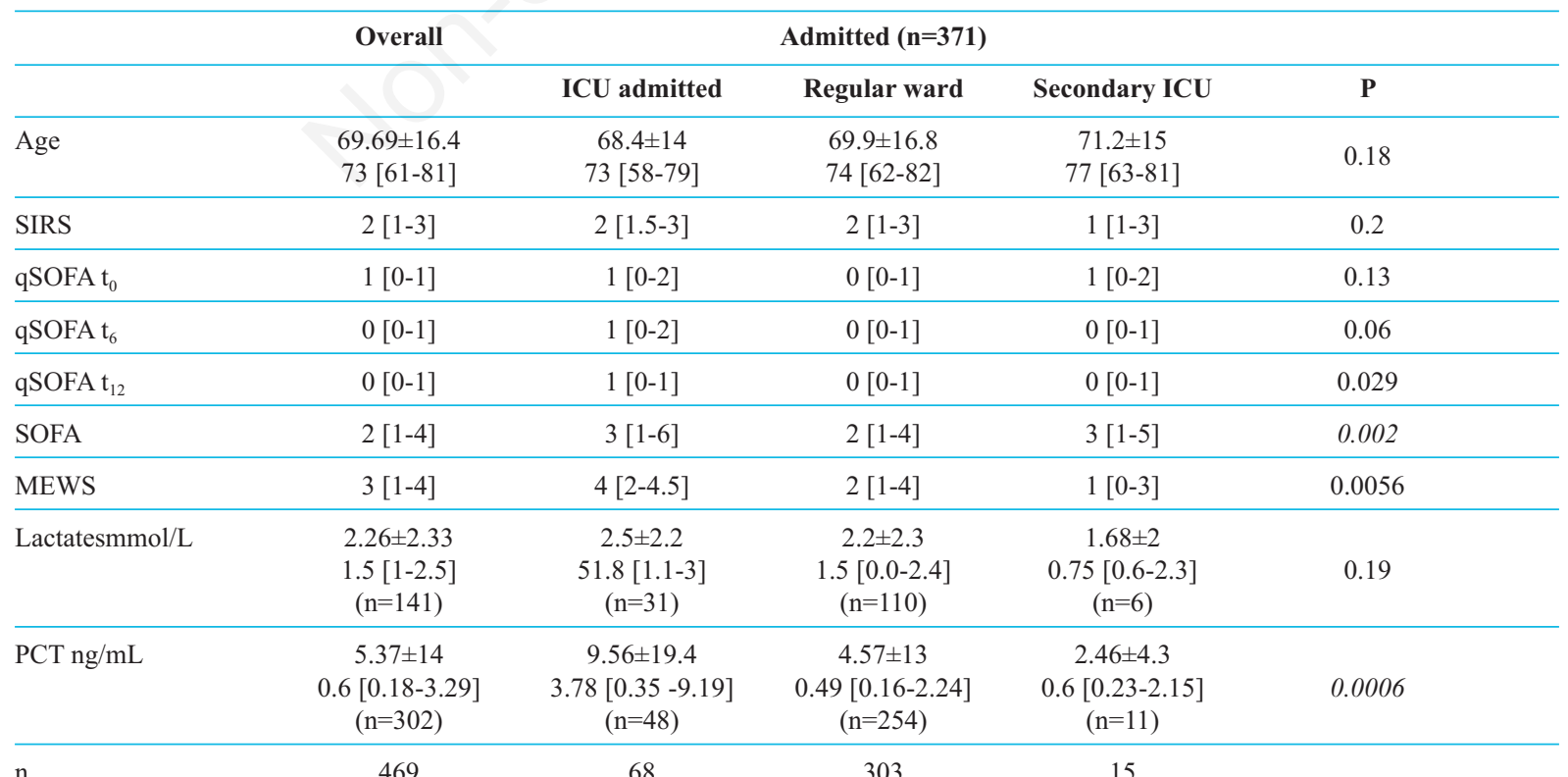

68

303

15

Comparisons were made according to Wilcoxon rank sum test for both primary and secondary ICU admission. $\mathrm{P}<0.05$ are in italic. Number of cases (n) in brackets. SIRS, systemic inflammatory response syndrome; SOFA, sequential organ failure assessment; qSOFA, quick SOFA; MEWS, modified early warning score; PCT, procalcitonin. 
explain the small differences in the performance of the scores in evaluating the two mortality outcomes; anyway, more data are needed to infer on this subject.

\section{Strengths and limitations}

The main strength of our study is its prospective nature, that was useful in reducing the risk of missing data that limited the validity of previous retrospective assessments of SIRS, qSOFA and SOFA.

Secondly, we calculate qSOFA three times rather than once as in previous studies. The repeated measurements of qSOFA followed the ideal characteristics of a screening tool for sepsis, where case discrimination needs to be a continuous process and cannot be decided by a single evaluation. This method was also useful to test the better timing interval for using qSOFA, a relatively new and still not routinely used tool.

Another strength is the inclusion of nearly all infected patients that arrived in our ED in a large complete dataset, not restricted to ICU patients, with a very low rate of drop out.

Moreover, this study was based on clinical data instead of administrative data and this real-world sample results were like other previous studies on the subject.

On the other hand, the first limitation is the definition of the study population. As there is no gold standard for defining sepsis, the study population was difficult to be determined. We included patients who visited the ED with a suspect of infection associated with signs of the SIRS, which could lead to a bias when evaluating and comparing SIRS with the other tools; we also included patients identified as septic according to clinical judgment.

Another limitation is that lactates and procalcitonin levels were assessed only in nearly half of patients, due to the relative novelty of the availability of the procalcitonin assay around the clock in our hospital and of the blood gas analyzer in our ED. The implementation of the protocol increased clinician awareness and in the final phase, the number of lactates and procalcitonin essays was higher.

Another limitation is the small number of patients admitted to the ICU, mainly due to the seniority of our population.

\section{Conclusions}

In our opinion, SOFA remains the most complete tool for the quick prognostic stratification and a more precise estimate of the individual risk assessment. SOFA, or alternatively qSOFA and lactates, in association with procalcitonin assessment could guide management decisions: to start early goal-directed therapy and to admit patients to a high level of care ward, if appropriate, with the aim to improve patient's outcome.

\section{References}

1. Singer M, Deutschman CS, Seymour CW, et al. The Third International Consensus Definitions for Sepsis and Septic Shock (Sepsis-3). JAMA 2016;315:801-10.

2. Bhattacharjee P, Edelson DP, Churpek MM. Identifying patients with sepsis on the hospital wards. Chest 2017;151:898-907.

3. Kaukonen KM, Bailey M, Pilcher D, et al. Systemic inflammatory response syndrome criteria in defining severe sepsis. N Engl J Med 2015;372:1629-38.

4. Vincent JL, Opal SM, Marshall JC, et al. Sepsis definitions: time for change. Lancet 2013;381:774-5.

5. Seymour CW, Liu VX, Iwashyna TJ, et al. Assessment of clinical criteria for sepsis: for the Third International Consensus Definitions for Sepsis and Septic Shock (Sepsis-3). JAMA 2016;315:762-74.

6. Song JU, Sin CK, Park HK, et al. Performance of the quick Sequential (sepsis-related) Organ Failure Assessment score as a prognostic tool in infected patients outside the intensive care unit: a systematic review and meta-analysis. Crit Care 2018;22:28.

7. Ramos JGR, da Hora Passos R, Teixeira MB, et al. Prognostic ability of quick-SOFA across different age groups of patients with suspected infection outside the intensive care unit: A cohort study. J Crit Care 2018;47:178-84.

8. Raith EP, Udy AA, Bailey M, et al. Australian and New Zealand Intensive Care Society (ANZICS) Centre for Outcomes and Resource Evaluation (CORE). Prognostic Accuracy of the SOFA Score, SIRS Criteria, and qSOFA Score for In-Hospital Mortality Among Adults With Suspected Infection Admitted to the Intensive Care Unit. JAMA 2017;317:290-300.

9. Bone RC, Balk RA, Cerra FB, et al. Definitions for Sepsis and Organ Failure and Guidelines for the Use of Innovative Therapies in Sepsis. Chest 1992;101:1644-55.

10. Subbe CP, Kruger M, Rutherford P, et al. Validation of a modified Early Warning Score in medical admissions. QJM 2001;94:521-6.

11. Shetty AL, Thompson K, Byth K, et al. Serum lactate cutoffs as a risk stratification tool for in-hospital adverse outcomes in emergency department patients screened for suspected sepsis. BMJ Open 2018;8:e15492.

12. Vijayan AL, Vanimaya, Ravindran S, et al. Procalcitonin: a promising diagnostic marker for sepsis and antibiotic therapy. J Intensive Care Internet 2017;5.

13. Szakmany T, Pugh R, Kopczynska M, et al. Defining sepsis on the wards: results of a multi-centre pointprevalence study comparing two sepsis definitions. Anaesthesia 2018;73:195-204.

14. Hwang SY, Jo IJ, Lee SU, et al. Low Accuracy of Positive qSOFA Criteria for Predicting 28-Day Mortality in Critically Ill Septic Patients During the Early Period After Emergency Department Presentation. Ann Emerg Med 2018;71:1.

15. Shapiro SS, Wilk MB. An analysis of variance test for normality (complete samples). Biometrika 1965;52:591611.

16. Wilcoxon F. Individual comparisons by ranking methods (PDF). Biometr Bull 1945;1:80-3.

17. Fisher RA. Statistical Methods for Research Workers. Oliver and Boyd; 1954. 
18. SAS Institute 2011. The SAS system for Windows. Release 9.2. SAS Inst., Cary, NC, USA.

19. R Core Team. R: A language and environment for statistical computing. R Foundation for Statistical Computing, Vienna, Austria; 2016. Available from: http:/www.R-project.org/

20. Jones AE, Trzeciak S, Kline JA. The Sequential Organ Failure Assessment score for predicting outcome in patients with severe sepsis and evidence of hypoperfusion at the time of emergency department presentation: Crit Care Med 2009;37:1649-54.

21. Rhee C, Dantes R, Epstein L, et al. Incidence and Trends of Sepsis in US Hospitals Using Clinical vs Claims Data 2009-2014. JAMA 2017; 318:1241.

22. Sakr Y, Rubatto Birri PN, Kotfis K, et al. Higher Fluid Balance Increases the Risk of Death From Sepsis: Results From a Large International Audit. Crit Care Med 2017;45:386-94.

23. Jung YT, Jeon J, Park JY, et al. Addition of lactic acid levels improves the accuracy of quick sequential organ failure assessment in predicting mortality in surgical patients with complicated intra-abdominal infections: a retrospective study. World J Emerg Surg 2018;13.

24. Henning DJ, Puskarich MA, Self WH, et al. An Emergency Department Validation of the SEP-3 Sepsis and
Septic Shock Definitions and Comparison With 1992 Consensus Definitions. Ann Emerg Med 2017;70:544552.e5.

25. van der Woude SW, van Doormaal FF, Hutten BA, et al. Classifying sepsis patients in the emergency department using SIRS, qSOFA or MEWS. Neth J Med 2018;76: 158-66.

26. Kim M, Ahn S, Kim WY, et al. Predictive performance of the quick Sequential Organ Failure Assessment score as a screening tool for sepsis, mortality, and intensive care unit admission in patients with febrile neutropenia. Support Care Cancer 2017;25:1557-62.

27. Maitra S, Som A, Bhattacharjee S. Accuracy of quick Sequential Organ Failure Assessment (qSOFA) score and systemic inflammatory response syndrome (SIRS) criteria for predicting mortality in hospitalized patients with suspected infection: a meta-analysis of observational studies. Clin Microbiol Infect 2018;24:1123-9.

28. Durusu Tanrı̈ver M, Halaçlı B, Sait B, et al. Daily surveillance with early warning scores help predict hospital mortality in medical wards. Turk J Med Sci 2016;46: 1786-91.

29. Abe EA. Gram-negative bacteremia induces greater magnitude of inflammatory response than Gram-positive bacteremia. Crit Care 2010;14:R27. 